J. Clin. Chem. Clin. Biochem.

Vol. 24, 1986, pp. $863-869$

(C) 1986 by Walter de Gruyter \& Co. Berlin - New York

\title{
Vergleichende quantitative Analytik klinisch-chemischer Kenngrößen im 24-Stunden-Urin und Morgenurin
}

Von M. Krieg und K.-J. Gunßer ${ }^{1}$ )

Institut für Klinische Chemie und Laboratoriumsmedizin, Berufsgenossenschaftliche Krankenanstalten „Bergmannsheil Bochum", Universitätsklinik Bochum

Elisabeth Steinhagen-Thiessen

Medizinische Klinik und

Hermann Becker

Urologische Klinik

Universitätskrankenhaus Hamburg-Eppendorf, Hamburg

(Eingegangen am December 19, 1985/May 29, 1986)

Zusammenfassung: An Hand von 10 klinisch-chemischen Kenngrößen wurde die Frage bearbeitet, welcher Zusammenhang zwischen der Morgenurin- und 24-Stunden-Ürinausscheidung besteht.

80 gesunde Probanden bildeten die Basis der Untersuchung. Diätetische Vorschriften brauchten während der Sammelperioden nicht eingehalten zu werden. Bezüglich des Morgenurins wurde lediglich gefordert, daß zwischen letzter Miktion und Morgenuringewinnung ein Zeitraum von 4 Stunden lag. Folgende Kenngrößen wurden bestimmt: Natrium, Kalium, Chlorid, Kreatinin, Harnstoff, Harnsäure, Glucose, Calcium, anorganischer Phosphor ünd Gesamteiweiß. Alle Kenngrößen bis auf Gesamteiweiß wurden vollmechanisiert ohne manuelle Vorverdünnung auf einem für Routinezwecke umgebauten Mehrkanalanalysator SMA 12/60 (Technicon) bestimmt.

Die Auswertung der Daten ergab:

(1) Die Streuung der Ausscheidungswerte ist im 24-Stunden-Urin im allgemeinen niedriger als im Morgenurin. Sie führt jedoch insgesamt zu relativ breiten Referenzintervallen.

(2) Fast durchgängig findet sich zwar ein signifikanter Zusammenhang $(p<0,01)$ zwischen den Ausscheidungen im 24-Stunden- und Morgenurin, der Korrelationskoeffizient liegt aber im Mittel bei nur 0,5.

(3) Die ermittelten Referenzintervālle (10/90-Perzentile) für die Konzentrationen der Kenngrößen im 24Stunden- und Morgenurin sind weitgehend kongruent.

Die Daten lassen vermuten, daß bei offensichtlich gesunden Probanden der Morgenurin die Ausscheidung über 24 Stunden ausreichend reflektiert. $\mathrm{Ob}$ dies bei pathologischen Zuständen zutrifft, muß noch gezeigt werden.

1) Diese Arbeit enthält wesentliche Teile der Doktorarbeit von K. J. G. 
Quantitative analysis of clinical chemical parameters in the $24 \mathrm{~h}$ urine and in the morning urine - A comparative studj'

Summary: Ten clinical chemical parameters were used to investigate the relationship between morning urine and 24-hour urine in 80 healthy probands.

During the period of the investigation there were no dietary restrictions. A period of at least 4 hours was required between the previous micturation and the collection of morning urine. The following parameters were determined: sodium, potassium, chloride, creatinine, urea, uric acid, glucose, calcium, inorganic phosphorus and total protein. Every parameter, with the exception of total protein, was determined without prior manual dilution, by a fully mechanized procedure, using a multichannel analyser SMA 12/60 (Technicon) adapted for routine purposes.

The data showed:

1) The scatter of the excretory values is generally lower in 24-hour urine than in morning urine, but collectively the reference intervals are relatively wide.

2) Almost without exception, there is a significant correlation between excretion in the 24-hour and morning urines, but the average correlation coefficient is only 0.5 .

3) The determined reference intervals ( $10 / 90$ percentile) for the concentrations of the parameters in 24-hour and morning urines are largely in agreement.

It is concluded from the data that the composition of morning urine of apparently healthy probands adequately reflects excretion of 24 hours. It remains to be seen whether this is also true for pathological states.

\section{Einführung}

Die Bestimmung von Natrium, Kalium, Chlorid, Kreatinin, Harnstoff, Harnsäure, Glucose, Calcium, anorganischem Phosphor und Gesamteiweiß im Urin spielt in einem klinisch-chemischen Laboratorium gegenüber der entsprechenden Serumanalytik quantitativ im allgemeinen eine untergeordnete Rolle. In einem größeren Klinikum mit mehreren Intensivstationen sowie größeren endokrinologischen, osteologischen, nephrologischen, onkologischen und urologischen Ambulanzen ist die Zahl der täglich indizierten Bestimmungen jedoch so hoch, daß eine vollmechanisierte Analytik zu erheblicher Zeiteinsparung führt. Aus diesem Grund haben wir erstmals einen Technicon SMA 12/60 so umgebaut, daß parallel und ohne manuelle Vorverdünnung 9 der oben genannten Kenngrößen quantitativ bestimmt werden können.

Die Leistungsfähigkeit dieses Systems veranlaßte uns, an Hand von 80 gesunden Probanden der Frage nachzugehen, welche Korrelationen bezüglich obengenannter klinisch-chemischer Kenngrößen zwischen 24-Stunden- und Morgenurin bestehen und wie sich die Referenzintervalle dieser Kenngrößen für beide Urine unterscheiden.

Vergleicht man die ermittelten Referenzintervalle der einzelnen auf Konzentrationsangaben basierenden Kenngrößen, so sind diese für den 24-Stunden- und Morgenurin weitgehend deckungsgleich. Wir meinen deshalb, daß trotz nur mäßiger Korrelationskoeffizienten, die im Mittel bei $r=0,5$ liegen, der Morgenurin bei einem offensichtlich gesunden Kollektiv die Ausscheidung über 24 Stunden widerspiegelt. $\mathrm{Ob}$ dies auch für pathologische Zustände zutrifft, muß noch gezeigt werden.

\section{Material und Methode}

Probanden

Mitarbeiter des Universitätskrankenhauses Hamburg-Eppendorf, die sich subjektiv gesund fühlten und voll arbeitsfähig waren, haben freiwillig an dieser Studie teilgenommen. Sie wurden über die Zielsetzung der Untersuchung unterrichtet und gebeten, einen Protokollbogen hinsichtlich Alter, Geschlecht, Körpergewicht, Körpergröße sowie Medikamenten- und Krankheitsanamnese auszufüllen. Die Sichtung der anamnestischen Angaben ergab keine Auffalligkeiten. Außerdem wurden Datum und Uhrzeit der 24-Stunden- und Morgenurin-Sammelperiode vermerkt. Bis auf wenige Ausnahmen erfolgten die Sammelperioden an zwei unmittelbar aufeinanderfolgenden Tagen. Bezüglich der Morgenurin-Sammelperiode wurde lediglich ein Mindestzeitraum von 4 Stunden zwischen letzter Miktion und der Morgenuringewinnung gefordert. Auflagen hinsichtlich Diät und Lebensführung während der Sammelperioden wurden nicht gemacht. Die Urine wurden in Kunststoffbehältern ohne Zusätze gesammelt und kühl gelagert. Im Labor erfolgte die Quantifizierung der gesamten Ausscheidungsmenge sowie die Bestimmung des spezifischen Gewichts mittels Aräometer. Neben der Uringewinnung erfolgte bei jedem Probanden zum gleichen Zeitpunkt eine Bluteñtnahme zur Bêstimmung unten genannter Kenngrößen im Serum mittels eines SMAC (Technicon). 


\section{Analyseverfahren}

Es wurde ein Technicon SMA 12/60 zum sogenannten SMA 9 U so umgebaut, daß erstmals parallel 9 Kenngrößen dieser Studie ohne Vorverdünnung der Urine vollmechanisiert bestimmt werden können. Lediglich Gesamteiweiß wurde gesondert manuell bestimmt. Die Nachweisverfahren lassen sich wie folgt kurz beschreiben:

\section{Natrium/Kalium}

Die Urinprobe wird einem luftsegmentierten Strom von $\mathrm{Li}$ thiumsulfat zugeführt und dann zur Enteiweißung gegen $\mathrm{H}_{2} \mathrm{O}$ dialysiert. Der segmentierte Empfängerstrom wird entlüftet und einem Flammenphotometer zugeführt. Untere Nachweisgrenze für Natrium: $12 \mathrm{mmol} / \mathrm{l}$; obere Nachweisgrenze: $380 \mathrm{mmol} / \mathrm{l}$. Untere Nachweisgrenze für Kalium: $4,5 \mathrm{mmol} / \mathrm{l}$; obere Nachweisgrenze: $150 \mathrm{mmol} / 1$.

\section{Chlorid}

Der Urinprobe wird Quecksilberthiocyanat zu- und dabei der Thiocyanatrest freigesetzt. Dieser reagiert mit Eisenionen und bildet einen roten $\mathrm{Fe}(\mathrm{CNS})_{3}-\mathrm{Komplex}$. Wellenlänge: $480 \mathrm{~nm}$. Untere Nachweisgrenze: $14 \mathrm{mmol} / \mathrm{l}$; obere Nachweisgrenze: 450 $\mathrm{mmol} / \mathrm{l}$.

\section{Kreatinin}

Die Urinprobe wird mit $18 \mathrm{~g} / \mathrm{l}$ wäßriger $\mathrm{NaCl}$-Lösung verdünnt und gegen $\mathrm{H}_{2} \mathrm{O}$ dialysiert. Nach der Dialyse wird eine 0,5 mol/1 NaOH-Lösung dem Strom zugeführt und anschließend Pikrinsäure hinzugegeben. Wellenlänge: $505 \mathrm{~nm}$. Untere Nachweisgrenze: $0,9 \mathrm{mmol} / \mathrm{l}$; obere Nachweisgrenze: $53 \mathrm{mmol} / \mathrm{l}$.

\section{Harnstoff}

Die Urinprobe wird mit $\mathrm{H}_{2} \mathrm{O}$ verdünnt, in einen Empfangsstrom von Diacetylmonoxim und Thiosemicarbazid dialysiert und schließlich mit $\mathrm{FeCl}_{3}$ versetzt. Das Gemisch wird auf $90^{\circ} \mathrm{C}$ erhitzt. Wellenlänge: $520 \mathrm{~nm}$. Untere Nachweisgrenze: 31,8 mmol/l; obere Nachweisgrenze: $988 \mathrm{mmol} / \mathrm{l}$.

\section{Harnsäure}

Die Urinprobe wird mit $\mathrm{NaOH}$ alkalisiert und in einen Empfängerstrom von Wolframat und Hydroxylamin dialysiert. Anschließend wird Phosphorwolframsäure zur Farbentwicklung zugegeben. Wellenlänge: $660 \mathrm{~nm}$. Untere Nachweisgrenze: 0,2 monol/l; obere Nachweisgrenze: $10 \mathrm{mmol} / \mathrm{l}$.

\section{Glucose}

Die Urinprobe wird mit $\mathrm{H}_{2} \mathrm{O}$ gemischt und gegen $\mathrm{H}_{2} \mathrm{O}$ dialysiert. Anschließend wird däs Reaktionsgemisch (Hexokinase) zugeführt. Wellenlänge: $340 \mathrm{~nm}$. Untere Nachweisgrenze: 0,3 mmol/1; obere Nachweisgrenze: $10 \mathrm{mmol} / \mathrm{l}$.

\section{Calcium}

Die Urinprobe wird mit $\mathrm{HCl}$ und 8-Hydroxychinolin gemischt und in eine Lösung von $\mathrm{HCl}$ und Cresolphthalein-Komplexon dialysiert. Nach der Dialyse wird alkalischer Puffer zugeführt. Wellenlänge: $570 \mathrm{~nm}$. Untere Nachweisgrenze: $0,3 \mathrm{mmol} / \mathrm{l}$; obere Nachweisgrenze: $10 \mathrm{mmol} / 1$.
Phosphor, anorg.

Die Urinprobe wird mit Schwefelsäure verdünnt und in Schwefelsäure dialysiert. Nach der Dialyse wird dem Strom Ammoniummolybdat und Chlorhydrazin zugeführt. Wellenlänge: 660 nm. Untere Nachweisgrenze: $1,5 \mathrm{mmol} / \mathrm{l}$; obere Nachweisgrenze: $50 \mathrm{mmol} / \mathrm{l}$.

\section{Gesamteiweiß}

$1 \mathrm{ml}$ Urin wird mit $200 \mu \mathrm{l}$ Trichloressigsäure versetzt, $15 \mathrm{~min}$ inkubiert und $10 \mathrm{~min}$ zentrifugiert. Der Niederschlag wird in $1 \mathrm{ml}$ Biuretreagenz aufgenommen und $30 \mathrm{~min}$ inkubiert. Abgelesen wird von einer Standardkurve (Protein-Standardlösung für Biuretmethode: $80 \mathrm{~g} / 1$ Albumin; Behringwerke). Wellenlänge: $540 \mathrm{~nm}$. Untere Nachweisgrenze: $5 \mathrm{mg} / \mathrm{l}$; obere Nachweisgrenze: $500 \mathrm{mg} / \mathrm{l}$.

Die Kalibrierung des SMA $9 \mathrm{U}$ erfolgt mit zwei wäßrigen, selbsthergestellten Standards.

Standard I enthält folgende Einwaagen ad $1000 \mathrm{ml}$ Aqua bidest. pH 5 (mit Eisessig eingestellt): $\mathrm{CaCl}_{2}: 1,1099 \mathrm{~g} ; \mathrm{Na}_{2} \mathrm{HPO}_{4}$. $2 \mathrm{H}_{2} \mathrm{O}: 8,8995 \mathrm{~g} ; D$-Glucose: $1,8016 \mathrm{~g}$; NaCl: $16,3632 \mathrm{~g} ; \mathrm{KCl}:$ $11,1840 \mathrm{~g}$; Kreatinin: 3,9592 g; Harnstoff: $60,0600 \mathrm{~g}$.

Standard II enthält folgende Einwaagen ad $987 \mathrm{ml}$ Aqua bidest. $+10 \mathrm{ml}$ Formaldehyd $(350 \mathrm{~g} / \mathrm{l})+3 \mathrm{ml}$ Essigsäure $(960 \mathrm{~g} / \mathrm{l})$ : Harnsäure: $1,6811 \mathrm{~g}$; Lithiumcarbonat: $0,6 \mathrm{~g}$.

Die Interassay-Variationskoeffizienten der SMA-Analysen sind mit zwei Urinpools an 9 aufeinanderfolgenden Tagen ermittelt worden und in Tabelle 1 zuisammengefaßt. Für das Gesamteiweiß ergaben sich diesbezüglich folgende Ergebnisse: Pool A: Konzentration: $20 \mathrm{mg} / \mathrm{l}, \mathrm{VK}=14,4 \%(\mathrm{n}=9)$; Pool B: Konzentration: $120 \mathrm{mg} / \mathrm{l}, \mathrm{VK}=10,2 \%(\mathrm{n}=9)$.

Tab. 1. Von-Tag-zu-Tag-Präzision $(V K ; n=9)$ der Nachweismethoden im Urin, die auf einem SMA 12/60 (Technicon) implementiert wurden.

\begin{tabular}{|c|c|c|c|c|c|}
\hline \multirow[t]{2}{*}{ Kenngröße } & \multirow[t]{2}{*}{ (Einheit) } & \multicolumn{2}{|c|}{ Urinpool A } & \multicolumn{2}{|c|}{ Urinpool B } \\
\hline & & $\overrightarrow{\mathbf{x}}$ & $\begin{array}{l}\text { VK } \\
(\%)\end{array}$ & $\overline{\mathbf{x}}$ & $\begin{array}{l}\text { VK } \\
(\%)\end{array}$ \\
\hline Natrium & $(\mathrm{mmol} / \mathrm{l})$ & 52 & 5,0 & 153 & 2,6 \\
\hline Kalium & $(\mathrm{mmol} / \mathrm{l})$ & 16 & 3,0 & 70 & 2,2 \\
\hline Chlorid & $(\mathrm{mmol} / \mathrm{l})$ & 52 & 3,2 & 182 & 1,6 \\
\hline Kreatinin & $(\mathrm{mmol} / \mathrm{l})$ & 2,65 & 3,3 & 14,1 & 1,8 \\
\hline Harnstoff & (mmol/l) & 141 & 7,5 & 293 & 5,7 \\
\hline Harnsäure & $(\mathrm{mmol} / \mathrm{l})$ & 1,21 & 2,6 & 3,62 & 2,7 \\
\hline Glucose & $(\mu \mathrm{mol} / \mathrm{l})$ & 466 & 9,5 & 5260 & 2,5 \\
\hline Calcium & $(\mathrm{mmol} / \mathrm{l})$ & 0,81 & 3,7 & 4,41 & 2,2 \\
\hline $\begin{array}{l}\text { Phosphor, } \\
\text { anorg. }\end{array}$ & $(\mathrm{mmol} / \mathrm{l})$ & 3,88 & 12,5 & 25,5 & 3,1 \\
\hline
\end{tabular}

\section{Statistik}

Die Daten wurden auf Lochkarten übertragen. Die statistischen Berechnungen wurden mit dem "Statistical Package for the Social Sciences" (SPSS) des Instituts für Mathematik und Datenverarbeitung in der Medizin der Universität Hamburg vorgenommen. Die Korrelationsberechnungen basieren auf Rangkorrelationen nach Spearman.

\section{Ergebnisse}

In Tabelle 2 sind Charakteristika des untersuchten Kollektivs aufgeführt. Die dargestellten Mittelwerte 
Tab. 2. Daten (Mittelwerte \pm Standardabweichungen) des untersuchten Kollektivs.

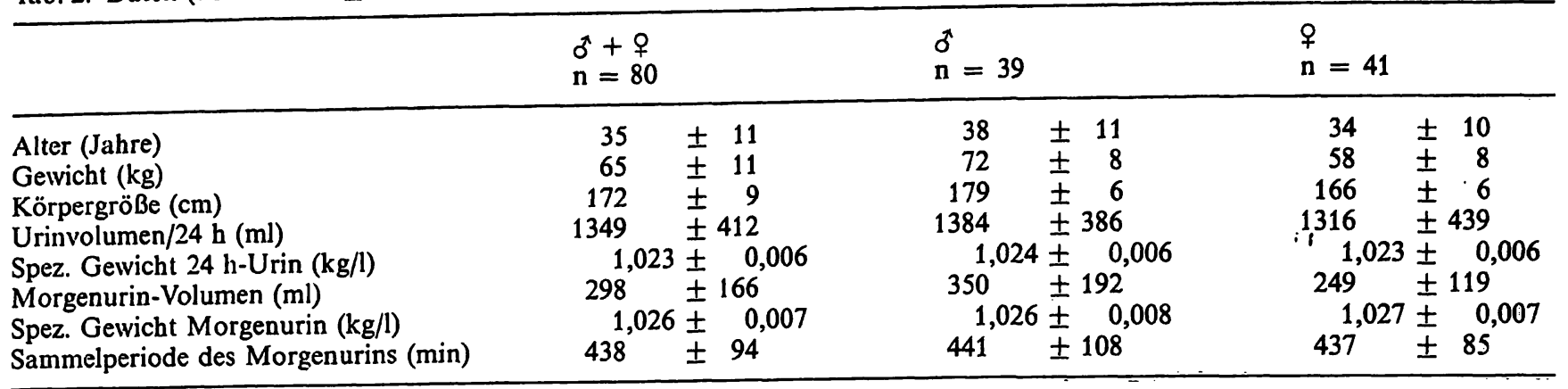

zeigen statistisch signifikante Geschlechtsunterschiede bezüglich Alter, Gewicht, Körpergröße und Morgenurinmenge. Da sich die Fragestellung primär auf Zusammenhänge zwischen 24-Stunden-Ausscheidung und Morgenurin konzentriert, wurde in den weiteren Betrachtungen vornehmlich das Gesamtkollektiv beschrieben. Die Konzentrationen der untersuchten Kenngrößen im Serum liegen bei allen Probanden im Referenzintervall. Sie bilden die in Tabelle 3 zusammengefaßten Mittelwerte und Standardabweichungen.

In Tabelle 4 sind die Ausscheidungsmengen, Konzentrationen und Variationskoeffizienten (VK) der 24Stunden-Urine den entsprechenden Daten des Morgenurins gegenüber gestellt. Naturgemäß müssen die Ausscheidungsmengen für alle Kenngrößen im 24Stunden-Urin deutlich höher liegen als im Morgenurin, wobei bemerkenswerterweise im Mittel für die meisten Kenngrößen etwa 25\% der Gesamtausscheidungsmenge im Morgenurin gefunden wird. Lediglich Kalium und Chlorid weichen hiervon mit einer mittleren Anteiligkeit der Morgenurin-Ausscheidungsmenge von unter $20 \%$ und anorg. Phosphor von über $30 \%$ ab. Da die Anteiligkeit der Morgenurin- ( $\bar{x}: 298$ $\mathrm{ml})$ an der 24-Stunden-Menge ( $\overline{\mathrm{x}}: 1349 \mathrm{ml})$ im Mittel bei $22 \%$ liegt, stimmen die mittleren Konzentrationen und Varianzen der Kenngrößen im 24-Stunden- und Morgenurin relativ gut überein. Geschlechtsspezifi-

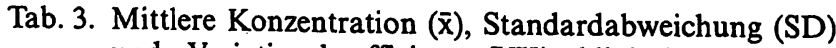
und Variationskoeffizient (VK) klinisch-chemischer Kenngrößen im Serum des untersuchten Kollektivs $(\mathrm{n}=80)$.

\begin{tabular}{llclc}
\hline Kenngröße (Konzentration) & $\overline{\mathrm{x}} \pm \mathrm{SD}$ & VK (\%) \\
\hline Natrium & $(\mathrm{mmol} / \mathrm{l})$ & 141 & \pm 2 & 0,7 \\
Kalium & $(\mathrm{mmol} / \mathrm{l})$ & $4,3 \pm 0,5$ & 11,6 \\
Chlorid & $(\mathrm{mmol} / \mathrm{l})$ & 106 & \pm 2 & 1,9 \\
Kreatinin & $(\mu \mathrm{mol} / \mathrm{l})$ & $88,4 \pm 17,7$ & 20,0 \\
Harnstoff & $(\mathrm{mmol} / \mathrm{l})$ & 458 & $\pm 1,06$ & 23,1 \\
Harnsäure & $(\mu \mathrm{mol} / \mathrm{l})$ & 256 & $\pm 1,4$ & 27,9 \\
Glucose & $(\mathrm{mmol} / \mathrm{l})$ & $5,11 \pm 0,944$ & 18,5 \\
Calcium & $(\mathrm{mmol} / \mathrm{l})$ & $2,37 \pm 0,08$ & 3,4 \\
Phosphor, anorg. & $(\mathrm{mmol} / \mathrm{l})$ & $1,16 \pm 0,19$ & 16,7 \\
Ges. Eiweiß & $(\mathrm{g} / \mathrm{l})$ & 69 & \pm 4 & 5,8 \\
\hline
\end{tabular}

sche signifikante Unterschiede finden sich in der Morgenurinmenge (bei fast gleicher durchschnittlicher Sammelperiode: männlich $441 \mathrm{~min}$; weiblich 437 min): Sie liegt bei Männern mit $350 \pm 192 \mathrm{ml}$ deutlich höher als bei Frauen mit $249 \pm 119 \mathrm{ml}$. Auch die mittlere Ausscheidungsmenge jeder einzelnen Kenngröße liegt bei Männern im Morgenurin signifikant höher.

Wie Tabelle 5 zeigt, ist die Anteiligkeit des Morgenurins am 24-Stunden-Urin erheblichen interindividuellen Schwankungen unterworfen. Dies ist sowohl an der Spanne zwischen minimaler und maximaler Anteiligkeit als auch an den Variationskoeffizienten erkennbar. Weiterhin ist hieraus ableitbar, daß im Morgenurin die einzelnen Kenngrößen in ganz unterschiedlicher Konzentration vorliegen müssen und keinesfalls gesetzmäßig im Morgenurin höher sind. Ergänzend sei erwähnt, daß die Anteiligkeit der Morgenurin-Werte an den Daten der 24-Stunden-Urine auch intraindividuell von Kenngröße zu Kenngröße z. T. stark variiert.

Um zu sehen, ob sich die VK's der Tabelle 4 minimieren lassen, indem die Meßwerte nicht als Menge oder Konzentration angegeben, sondern zusätzlich auf Variable wie ausgeschiedene Kreatininmenge, Körperoberfläche etc. bezogen werden, sind in Tabelle 6 verschiedene Verrechnungen mit den daraus resultierenden VK's zusammengefật. Eine für alle KenngröBen gültige Minimierung der interindividuellen Varianz läßt sich nicht erreichen, wenn auch $z$. B. die VK's der Ausscheidung von Harnstoff und Glucose im 24-Stunden-Urin bei Kreatininbezug sowie von Harnsäure und Calcium in 24-Stunden-Urin bei Bezug auf Körperoberfläche vergleichsweise deutlich kleiner sind.

Korreliert man die jeweiligen Daten der Morgen- und 24-Stunden-Urine, so finden sich nur Koeffizienten zwischen 0,323 und 0,711, wobei der mittlere Koeffizient am höchsten ist $(r=0,588)$, wenn alle Werte auf die Kreatininausscheidung bežogén werden. Die Signifikanzberechnung ergibt in den meisten Fällen ein $\mathrm{p}<0,01$. 


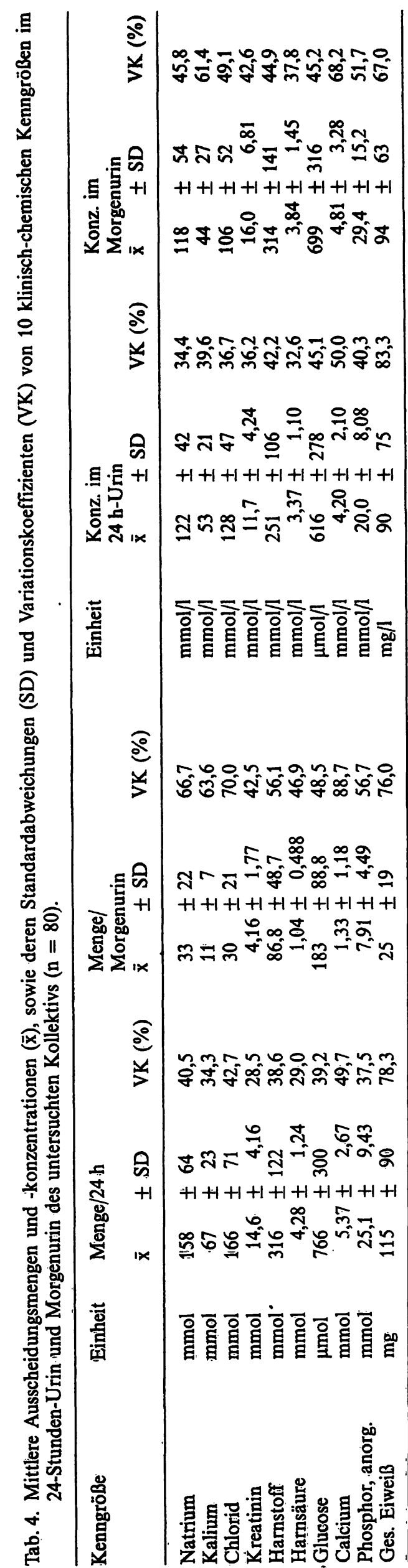

Tab. 5. Minimum (Min.), Maximum (Max.) und Variationskoeffizient (VK) des prozentualen Anteils der Morgenurin- an den 24-Stunden-Urin-Daten $(n=80)$. Die Anteiligkeit hinsichtlich der klinisch-chemischen Kenngrößen bezieht sich auf Mengenangaben.

\begin{tabular}{llll}
\hline Kenngröße & Min. (\%) & Max. (\%) & VK (\%) \\
\hline Zeitspanne & 16 & 50 & 21,7 \\
Urinausscheidung & 6 & 62 & 50,0 \\
Natrium & 4 & 50 & 51,8 \\
Kalium & 3 & 60 & 60,0 \\
Chlorid & 4 & 44 & 48,3 \\
Kreatinin & 3 & 58 & 38,1 \\
Harnstoff & 3 & 64 & 43,5 \\
Harnsäure & 4 & 60 & 40,8 \\
Glucose & 4 & 65 & 46,7 \\
Calcium & 4 & 71 & 55,1 \\
Phosphor, anorg. & 2 & 78 & 48,3 \\
Ges. Eiweiß & 2 & 89 & 101,3 \\
\hline
\end{tabular}

In Tabelle 7 sind für die 10 untersuchten Kenngrößen jeweils Referenzintervalle für 24-Stunden- und Morgenurin berechnet. Es ist offensichtlich, daß bei der Angabe der Daten als Konzentration die Intervalle für den Morgenurin durchgängig breiter sind als für den 24-Stunden-Urin. Andererseits ist aber eine sehr weitgehende Überlappung gegeben. Auch bei den anderen von uns gewählten Bezugsgrößen sind die Referenzintervalle des Morgenurins erwartungsgemäß breiter. Vorteile gegenüber den dargestellten Referenzintervallen sind nicht erkennbar. Signifikante Geschlechtsunterschiede fehlen, wenn die Ausscheidungswerte als Konzentrationen angegeben werden. Eine Tendenz zu höheren Werten bei Männern ist jedoch erkennbar.

\section{Diskussion}

Die Umrüstung eines etwa 10 Jahre alten und bislang für Serumanalysen benutzten SMA $12 / 60$ in einen Mehrkanalanalysator für Urinproben, den wir als SMA $9 U$ bezeichnen, erwies sich als technisch problemlos, hinsichtlich der analytischen Präzision als akzeptabel (Tab. 1) und für die täglich anfallende quantitative Urindiagnostik als eminent hilfreich. Sie hat diesen Arbeitsplatz mit täglich etwa 50 Primärproben bezüglich des Arbeitsaufwandes ungefähr halbiert. Für ein Klinikum der Maximalversorgung mit zentralisierter klinisch-chemischer Diagnostik kann deshalb die Umrüstung eines möglicherweise zur Ausmusterung anstehenden SMA 12/60 uneingeschränkt empfohlen werden.

Neben dem praktischen Nutzen hat dieses leistungsfähige System den Vorteil, Probleme anzugehen, die wegen des bisher notwendigen analytischen Aufwandes nicht oder nur fragmentarisch abgehandelt wurden. So wird in der vorliegenden Studie erstmals 


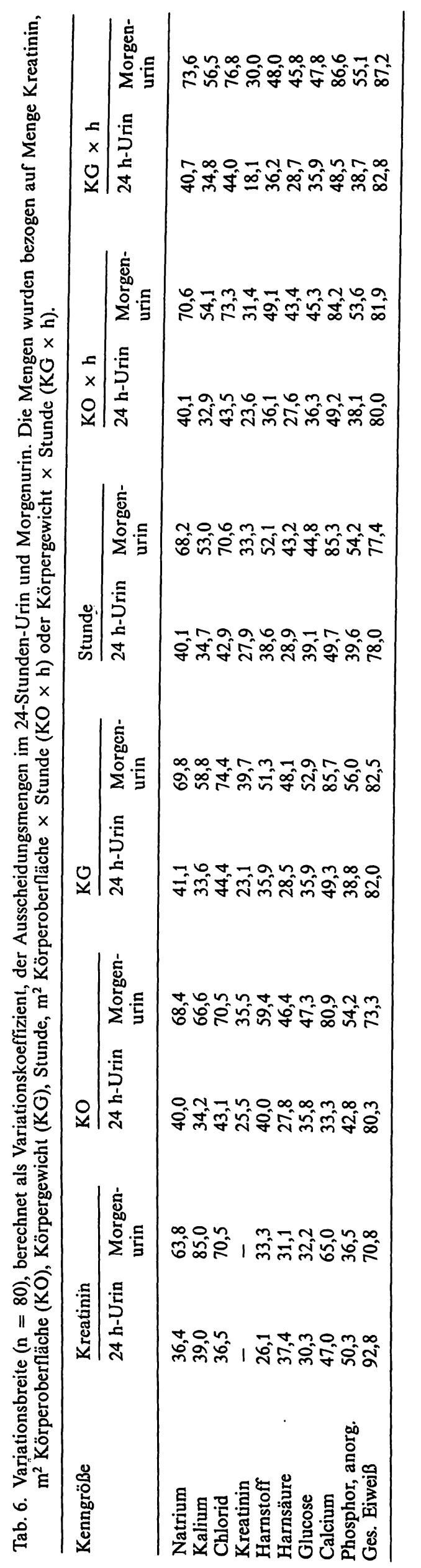

Tab. 7. Referenzintervalle (10/90-Perzentile) der Konzentrationen im 24-Stunden-Urin und Morgenurin, ermittelt an einem Kollektiv gesunder Probanden $(n=80)$.

\begin{tabular}{|c|c|c|c|c|c|}
\hline \multirow{2}{*}{ Kenngröße } & \multirow{2}{*}{$\begin{array}{l}\text { Einheit } \\
\mathrm{mmol} / \mathrm{l}\end{array}$} & \multicolumn{2}{|c|}{$24 \mathrm{~h}$-Urin } & \multicolumn{2}{|c|}{ Morgenurin } \\
\hline & & 71 & -171 & 54 & -190 \\
\hline Kalium & $\mathrm{mmol} / 1$ & 32 & -83 & 20 & -80 \\
\hline Chlorid & $\mathrm{mmol} / \mathrm{l}$ & 75 & -199 & 46 & -168 \\
\hline Kreatinin & $\mathrm{mmol} / \mathrm{l}$ & 7,07 & $7-16,8^{\prime}$ & 7,96 & $6-\quad 26,5$ \\
\hline Harnstoff & $\mathrm{mmol} / \mathrm{l}$ & 141 & -388 & 141 & $\div 494$ \\
\hline Harnsäure & $\mathrm{mmol} / 1$ & 2,28 & $3-\quad 4,75$ & 2,18 & $3-\quad 5,47$ \\
\hline Glucose & $\mu \mathrm{mol} / 1$ & 316 & -960 & 339 & -1130 \\
\hline Calcium & $\mathrm{mmol} / 1$ & 1,6 & $-7,0$ & 1,3 & $-\quad 8,9$ \\
\hline $\begin{array}{l}\text { Phosphor, } \\
\text { anorg. }\end{array}$ & $\mathrm{mmol} / 1$ & 10 & -31 & 13 & -44 \\
\hline Ges. Eiweiß & $\mathrm{mg} / \mathrm{l}$ & 30 & -150 & 40 & -150 \\
\hline
\end{tabular}

an gesunden Probanden ein systematischer Vergleich zwischen 24-Stunden- und Morgenurin durchgeführt. In der Vergangenheit ist solch ein Vergleich nur vereinzelt erfolgt, beispielsweise bezüglich angeborener Aminosäurestoffwechselstörungen (1), der Purinstoffwechselstörungen (2), der Proteinurien $(3,4)$, der Kaliumausscheidung (5), der Nebennierenrindenfunktion (6) und der. Überwachung der Chloridausscheidung (7).

Unsere Ergebnisse lassen sich wie folgt interpretieren:

(1) Die mittlere Zeitspanne der Morgenurinsammlung betrug 7,3 Stunden. Dies entspricht auf 24 Stunden bezogen einem Zeitanteil von 30\%. Dagegen betrug der prozentuale Anteil der Morgenurin- an der 24-Stunden-Menge nur $22 \%$, womit tendenziell die von anderer Seite $(8,9)$ gemachte Beobachtung bestätigt wird, daß in der zweiten Nachthälfte ein zirkadianes Minimum der Urinausscheidungsmenge besteht. Dieses Ausscheidungsminimum findet sich im Mittel auch für die Mehrzahl der untersuchten Kenngrößen, insbesondere aber bei Chlorid, Kalium und Natrium mit Morgenurin-Anteilen von $16-21 \%$ an der 24-Stunden-Menge. Kreatinin scheint über den Tag fast gleichmäßig ausgeschieden zu werden, da der mittlere Mengenanteil im Morgenurin von $29 \%$ fast identisch ist mit dem oben erwähnten Zeitanteil von $30 \%$. Die Phosphorausscheidung schließlich zeigt in den Nachtstunden eine Tendenz zu höheren Werten, da im Morgenurin im Mittel eine Ausscheidungsanteiligkeit von $32 \%$ gegenüber der 24-Stunden-Ausscheidungsmenge gefunden wurde. $\mathrm{Zu}$ ähnlichen Aussagen kommen Mertz (8) sowie Vahlensieck et al. (9). Ob diese diskrete Rhythmik die Anteiligkeit der Morgenurinausscheidung an der 24-Stunden-Menge kollektivmäßig beeinflußt, mag bezweifelt werden, wenn man die hohen Standardabweichungen und Variationskoeffizienten der Tabelle 4 berücksichtigt. So sind die Ausscheidungsmuster nicht nur von Proband zu Proband unterschiedlich, sondern auch das Ver- 
hältnis der Kenngrößen zueinander unterliegt keiner erkennbaren Gesetzmäßigkeit. Insgesamt tendieren wir deshalb zu der Feststellung, daß sich im Mittel der Morgenurin bei einer vorgegebenen Sammelperiode von mindestens 4 Stunden zeitlich und mengenmäßig in einer etwa gleichen prozentualen Anteiligkeit zum 24-Stunden-Urin einpendelt. Diese Feststellung wird unterstützt durch die weitgehend kongruenten 10/90-Perzentilbereiche (Tab. 7) der Konzentrationen im 24-Stunden-Urin und Morgenurin.

(2) Unsere Daten zeigen, daß mit Ausnahme von Gesamteiweiß die interindividuelle Streuung der Ausscheidungswerte in den Morgenurinen durchgängig größer ist als in den 24-Stunden-Sammelurinen. Dieses Phänomen ist nicht nur auf die interindividuelle Streuung der Morgenurin-Sammelperiode mit einem VK von $21 \%$ zurückzuführen. Die auf eine Stunde bezogenen Ausscheidungswerte der Morgenurine zeigen zwar beispielsweise für Kalium und Kreatinin deutlich kleinere Streuungen, insgesamt aber ist die interindividuelle Streuung der Morgenurinwerte auch bei dieser Verrechnung regelmäßig größer als die der 24-Stunden-Sammelurinwerte. Gleiches gilt, wenn andere Verrechnungen, wie beispielsweise der Bezug der Ausscheidungsmenge auf die Kreatininausscheidung, auf das Körpergewicht und/oder die Körperoberfläche vorgenommen wurden. Das Ausscheidungsverhalten scheint demnach von endogenen und exogenen Faktoren bestimmt zu sein, die über 24 Stunden individuell ganz unterschiedlich zum Tragen kommen und deshalb bei verkürzten Sammelperioden fast gesetzmäßig zu größeren Varianzen der Ausscheidungsdaten führen müssen. Warum die Eiweißausscheidung dieser Gesetzmäßigkeit nicht folgt, bleibt derzeit ungeklärt.

Aber auch bezüglich der 24-Stunden-Urine ist die interindividuelle Varianz für einzelne Kenngrößen sehr groß, wenn man sie mit denen des Serums (Tab. 5) vergleicht. Bemerkenswerterweise zeichnet sich

\section{Literatur}

1. Tsai, M. Y., Marsihall, J. G. \& Josephson, M. W. (1980) Clin. Chem. 26, 1804-1808.

2. Kaufmann, J. M., Green, M. L. \& Seegmiller, J. E. (1968) J. Pediat. 73, 583-592

3. Ginsberg, J. M., Chang, B. S., Matrese, R. A. \& Garella, S. (1983) N. Engl. J. Med. 309, 1543-1546.

4. Shaw, A. B., Ridson, P. \& Lewis-Jackson, J. D. (1983) Brit. Med. J. 287, $929-932$.

5. Nordin, B. E. C. (1959) Lancet $i i, 368-371$.

6. Walker, M. S. (1977) Ann. Clin. Biochem. 14, 203-206.

7. Kaplan, N. M., Simmon, S. M., Phee, Mc C., Carnegie, A., Stefann, C. \& Cade, S. (1982) Arch. Int. Med. 142, $1638-1641$.

8. Mertz, D. P. (1964) Dtsch. Med. Wochenschr. 89, 23272332.

9. Vahlensieck, E. W., Bach, D. \& Hesse, E. (1982) Urol. Res. $10,195-203$. eine negative Korrelation ab, d.h. bei extrem niedrigen VK's im Serum (z. B. Natrium, Chlorid, Calcium) sind die entsprechenden VK's im Urin relativ hoch, und umgekehrt (z. B. Harnsäure). Dieser Zusammenhang ist verständlich, da der menschliche Organismus das schwankende alimentäre Angebot um so massiver über die Niere regulieren muß, je enger er den Konzentrationsbereich im Serum fassen muß. Hieraus darf abgeleitet werden, da $B$ unter nicht standardisierten alimentären Bedingungen auch die 24-StundenUrine relativ große, für diagnostische Fragestellungen möglicherweise unbefriedigende Referenzintervalle besitzen.

(3) Der Zusammenhang zwischen Morgen- und 24Stunden-Urin muß aufgrund der Korrelationskoeffizienten als nicht sehr eng angesehen werden, so da $B$ aus dieser Studie eine Ersetzbarkeit des 24-StundenUrins durch den Morgenurin nicht zwingend abgeleitet werden kann. Wir glauben trotzdem, daß der Morgenurin bei einem gesunden Kollektiv die Ausscheidungsverhältnisse über 24 Stunden in erster Näherung widerspiegelt und er demnach dem 24-Stunden-Urin zumindest im Rahmen einer orientierenden Untersuchung gleichwertig zur Seite gestellt werden kann. Seine Daten sollten als Konzentrationen angegeben werden, da diese Verrechnung am praktikabelsten ist. Die als Konzentrationen angegebenen Referenzintervalle des Morgenurins (Tab. 7) sind mit den von uns in dieser Studie ermittelten Referenzintervallen des 24-Stunden-Urins weitgehend kongruent, wobei die Daten des 24-Stunden-Urins wiederum von Angaben der Literatur $(10,11,12)$ nicht stärker abweichen als die Literaturangaben untereinander. Um eine weitere Minimierung der relativ großen Varianz zu erreichen, wäre in erster Linie eine Standardisierung der alimentären Situation vor der Urinsammlung notwendig, wobei diese Standardisierung dem diagnostischen Stellenwert des Morgenurins ebenso zugute käme wie dem des 24-Stunden-Urins.

10. Geigy Scientific Tables (1981) (Lentner, C., ed.), Vol. 1, Ciba-Geigy Ltd., Basel.

11. Plenert, W. \& Heine, W. (1984) Normalwerte, 6. Auflage, Karger, Basel.

12. Gunßer, K. J. (1985) Kann die Untersuchung des Morgenurins in der klinisch-chemischen Diagnostik die Untersuchung des 24-Stunden-Urins ersetzen? Medizinische Dissertation, Universität Hamburg.

Prof. Dr. med. M. Krieg

Institut für Klinische Chemie und Laboratoriumsmedizin

Bergmannsheil Bochum - Universitätsklinik

Hunscheidtstr. 1

D.4630 Bochum 1 
